\title{
RETRACTED ARTICLE: Evaluation of intestinal parasites among referred patients: report of 4637 cases
}

\author{
Ali Asgari ${ }^{1}$ - Mohammad Davood Sharifi ${ }^{2}$ Hosein Amini Anabad ${ }^{3}$. \\ Amin Zamani Nughabi ${ }^{4} \cdot$ Amir Farshid Fayyaz $^{5} \cdot$ Ali Akbar Jafari ${ }^{6}$
}

Received: 22 April 2015/ Accepted: 4 June 2015/Published online: 14 June 2015

(C) Indian Society for Parasitology 2016

This article has been retracted at the request of the Editorin-Chief, The Indian Society for Parasitology (ISP) and the Publisher per the Committee on Publication Ethics guidelines. The article shows evidence of irregularities in authorship, there is strong reason to believe that the peer review process was compromised and the authors have plagiarized parts from the following articles:

Mohammad Sabzikarian, Saeideh Najafi, Hosein Amini Anabad, Vahid Behnod, Emad Yahaghi, Koorosh Ahmadi, Frequency of intestinal protozoan parasites in patients with gastrointestinal disorders in south of Karaj, Iran. Journal of Parasitic Diseases. DOI: 10.1007/s12639-015-0703-z.

H. Sadeghi, M. Bakht, H. Saghafi, T. Shahsavari, Prevalence of intestinal parasites in a population in Eghbalieh city from Qazvin Province, Iran. Journal of Parasitic Diseases. 2015; 39:2 126-129 DOI: 10.1007/s12639-013-0366-6.

As such the validity of the content of this article cannot be verified.

The online version of this article contains the full text of the retracted article as electronic supplementary material.

Electronic supplementary material The online version of this article (doi:10.1007/s12639-015-0698-5) contains supplementary material, which is available to authorized users.

Ali Akbar Jafari

a.jafary63@yahoo.com

1 Department of Infectious Diseases, AJA University of Medical Sciences, Tehran, Iran

2 Department of Emergency Medicine, Imam Reza Hospital, Faculty of Medicine, Mashhad University of Medical Sciences, Mashhad, Iran

3 Health Management Research Center, Baqiyatallah University of Medical Sciences, Tehran, Iran

4 Resident of Emergency Medicine, Mashhad University of Medical Sciences, Mashhad, Iran

5 Department of Legal Medicine, AJA University of Medical Sciences, Tehran, Iran

6 Young Researchers and Elite Club, Parand Branch, Islamic Azad University, Parand, Iran 